\title{
Neue Prävalenzdaten vom KiGGS
}

m September letztes Jahres veröffentlichte das Robert-Koch-Institut in Berlin erste Auswertungen des bundesweiten Kinder- und Jugendgesundheitssurveys (KiGGS). In die Erfassung waren in den Jahren 2003 bis 2006 Daten aus einer bevölkerungsbezogenen Stichprobe von 17.641 Kindern und Jugendlichen bis 17 Jahre aus 167 Orten Deutschlands aufgenommen worden. Angaben zu körperlichen Beschwerden und Befindlichkeit sowie akuten und chronischen Krankheiten, darunter auch Allergien, wurden erhoben (Bundesgesundheitsblatt Gesundheitsforschung Gesundheitsschutz 2006; 49: 1050-8 oder www.kiggs.de).

Die Ergebnisse zeigen, dass 16,7\% aller deutschen Kinder und Jugendlichen an einer allergischen Erkrankung leiden,
Jungen sind mit 18,0\% häufiger betroffen als Mädchen mit 15,4\%. Dabei sind $8,9 \%$ an Heuschnupfen erkrankt (Jungen 10,3\%, Mädchen 7,5\%). Erwartungsgemäß nimmt die Häufigkeit von Allergien mit steigendem Alter zu: von unter $1 \%$ auf über $15 \%$. Die Prävalenz ist bei Kindern und Jugendlichen mit Migrationshintergrund niedriger (13\% vs. $17,6 \%$ ), ebenso bei Kindern aus sozial schwachen Verhältnissen. Kinder aus Ost- und Westdeutschland sind inzwischen fast gleich häufig betroffen.

Abb. 1 nennt die Allergene, die am häufigsten Sensibilisierungen hervorrufen. Insgesamt 40,8\% der Kinder und Jugendlichen zeigen eine IgE-vermittelte Sensibilisierung gegen mindestens ein Allergen. Jungen $(45,0 \%)$ sind häufiger

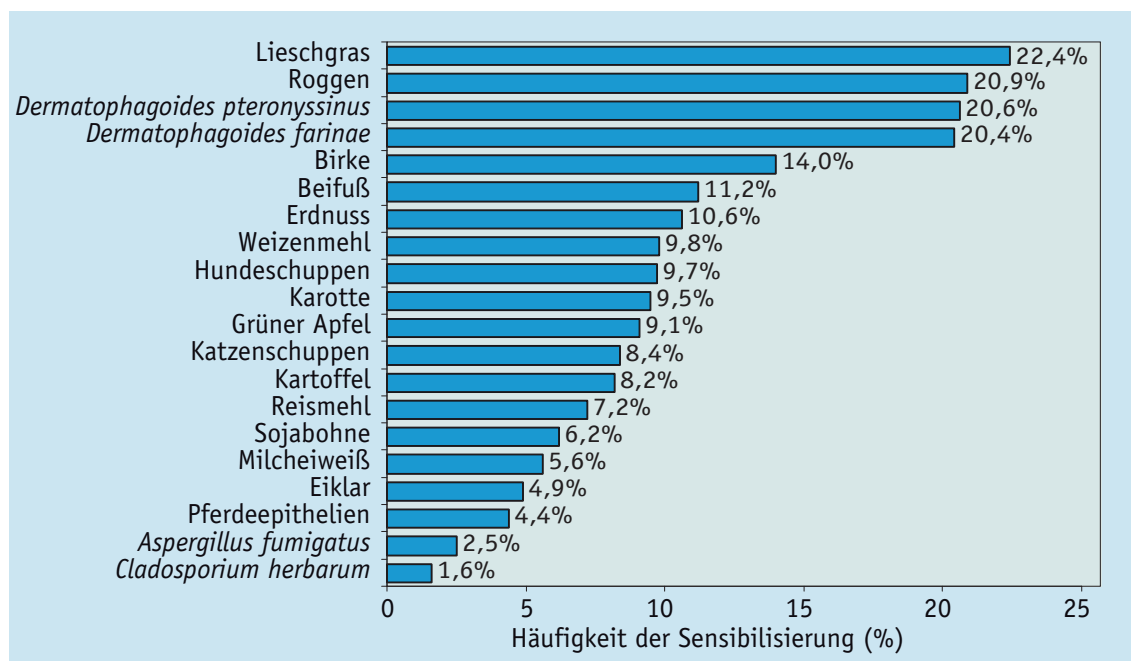

Abbildung 1. Hitliste der IgE-vermittelten Sensibilisierungen

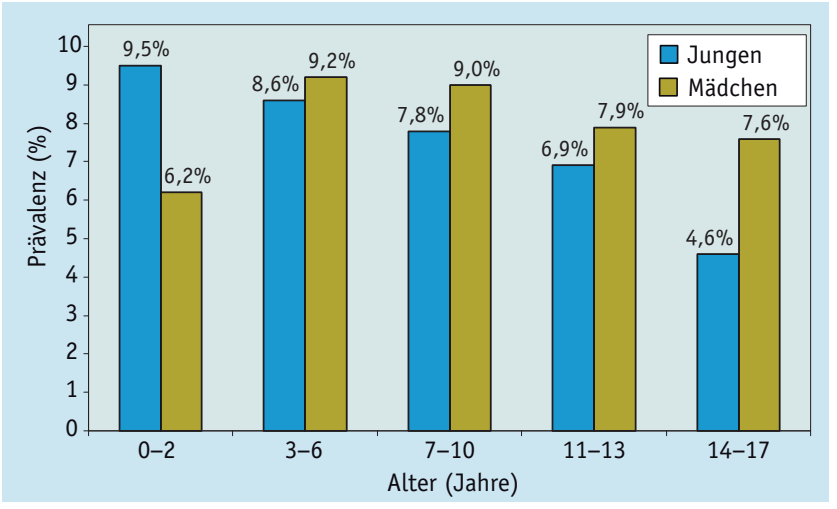

Abbildung 2. Prävalenz des atopischen Ekzems
Impressum „GPA-Nachrichten“

Verantwortlich für den Inhalt

Dr. Ernst Rietschel, Köln

Redaktion

Markus Seidl

Verlag

(๔) Urban \& Vogel GmbH, München

\section{GPA-Geschäftsstelle}

Dr. Frank Friedrichs, Aachen

Tel.: (02 41) 9800486

Fax: (02 41) 9800259

E-Mail:info@gpaev.de

sensibilisiert als Mädchen (36,4\%). Gegen inhalative Allergene haben 37,2\% spezifische IgE-Antikörper, gegenüber Nahrungsmitteln 20,2\%, wobei als häufigstes Nahrungsmittelallergen die Erdnuss in 10,6\% der Fälle gefunden wird.

An einem atopischen Ekzem sind $7,7 \%$ der Kinder und Jugendlichen in allen Altersgruppen erkrankt ohne Unterschied zwischen Mädchen und Jungen. Abb. 2 fasst die Prävalenz des atopischen Ekzems in den unterschiedlichen Altersgruppen zusammen. Die Lebenszeitprävalenz des allergischen Kontaktekzems liegt bei 9,9\%, wobei Mädchen mit $13,8 \%$ häufiger betroffen sind als Jungen $(6,2 \%)$. Nur hier findet sich ein eindeutiger Unterschied zwischen den alten $(10,2 \%)$ und den neuen $(8,4 \%)$ Bundesländern. An Asthma leiden 3,0\%, wobei auch hier Jungen mit 3,5\% häufiger betroffen sind als Mädchen mit 2,5\% (Abb. 3). In ländlichen Gebieten ist Asthma seltener als in der Stadt. 